\title{
Perinatal pathology reports: A guide for obstetricians
}

J. Ciaran

\section{Neil J. Sebire (Please indicate Neil Sebire as the corresponding author.)}

J. Ciaran Hutchinson MBBS PhD FRCPath Department of Histopathology, Great Ormond Street Hospital for Children NHS Foundation Trust, London, UK. Conflicts of interest: none declared

Neil J. Sebire MD FRCPath University College London Great Ormond Street Institute of Child Health, London, UK. Conflicts of interest: none declared

Abstract

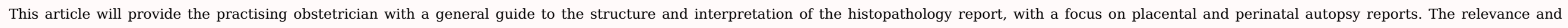

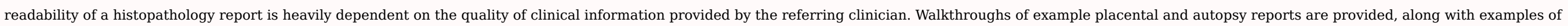
pathologies and their possible significance to the underlying diagnosis.

Keywords: autopsy; pathology; perinatal; placenta

\section{Introduction}

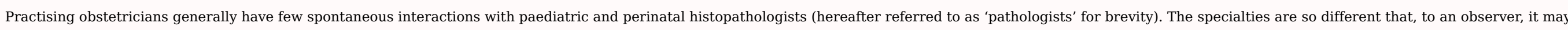

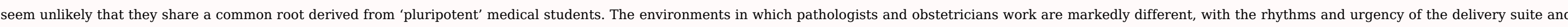

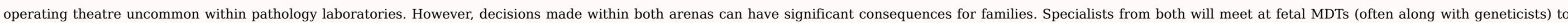

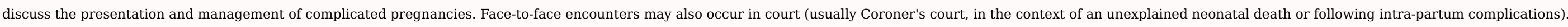

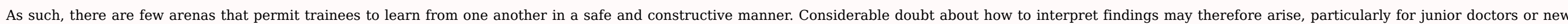

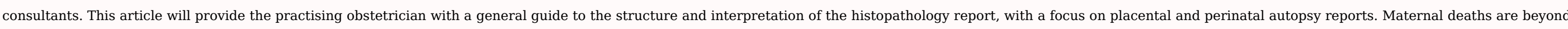

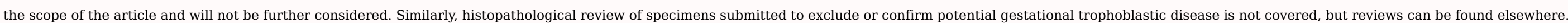

The examples are based on real cases but have been altered to protect patient confidentiality.

\section{Generating a useful pathology report}

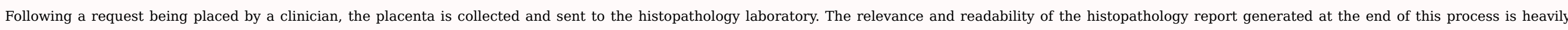

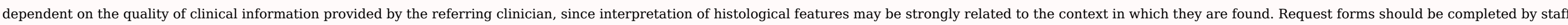

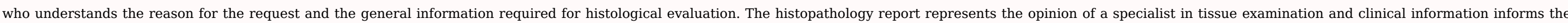

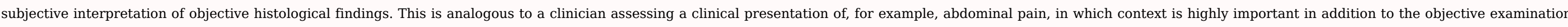

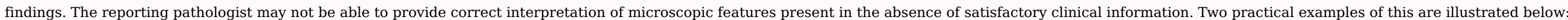

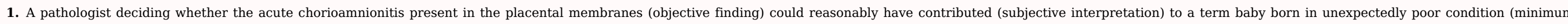

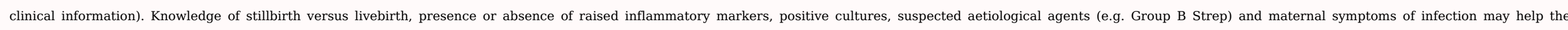
pathologist to provide a clinically useful comment in this case.

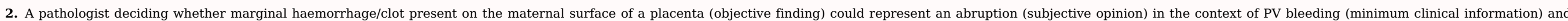




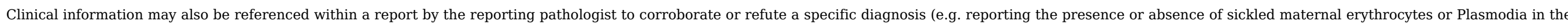
assessment of placental histology of a feverish woman of East African origin).

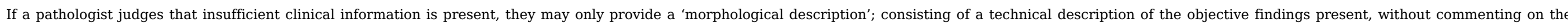

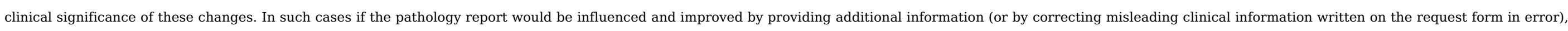
contacting the pathologist in question will afford them the opportunity to provide a more meaningful report in an addendum.

\section{Interpretation of a placental pathology report}

\section{The structure of a placental pathology report: clinical details and supporting information}

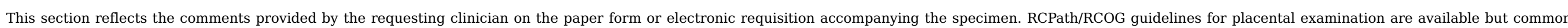
clinical indications for pathological examination of the singleton placenta include:

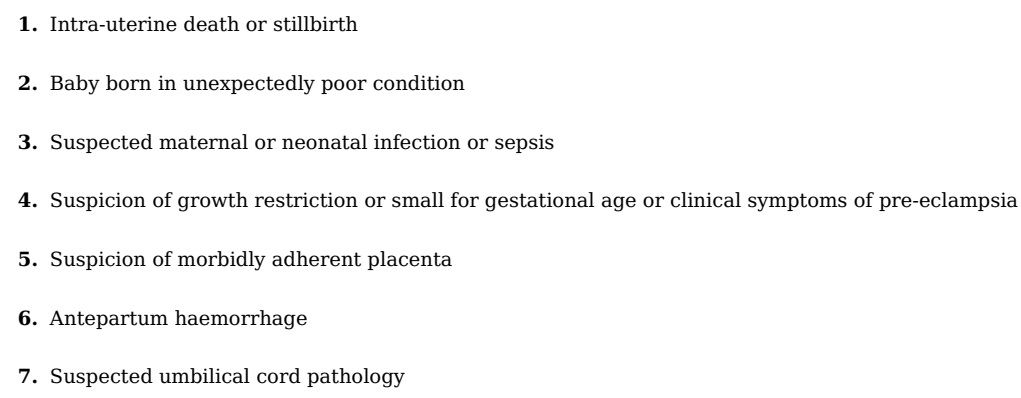

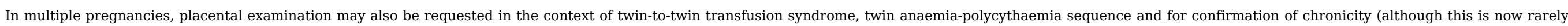

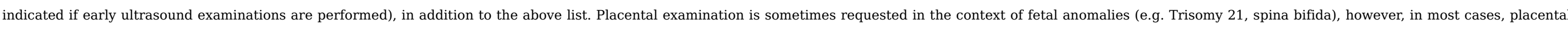
examination adds little useful additional clinical information in the context of a confirmed anomaly and few fetal anomalies have specific associated placental abnormalities.

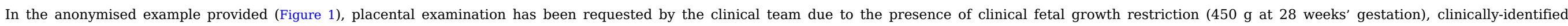

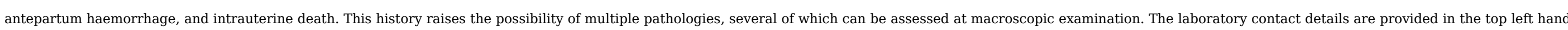

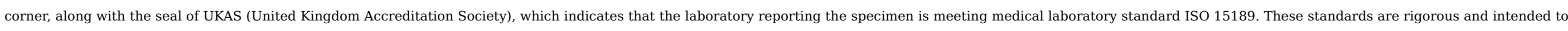
ensure quality and competence within diagnostic services. Main UKAS inspections are generally performed every three years, with interim inspections occurring more frequently. 


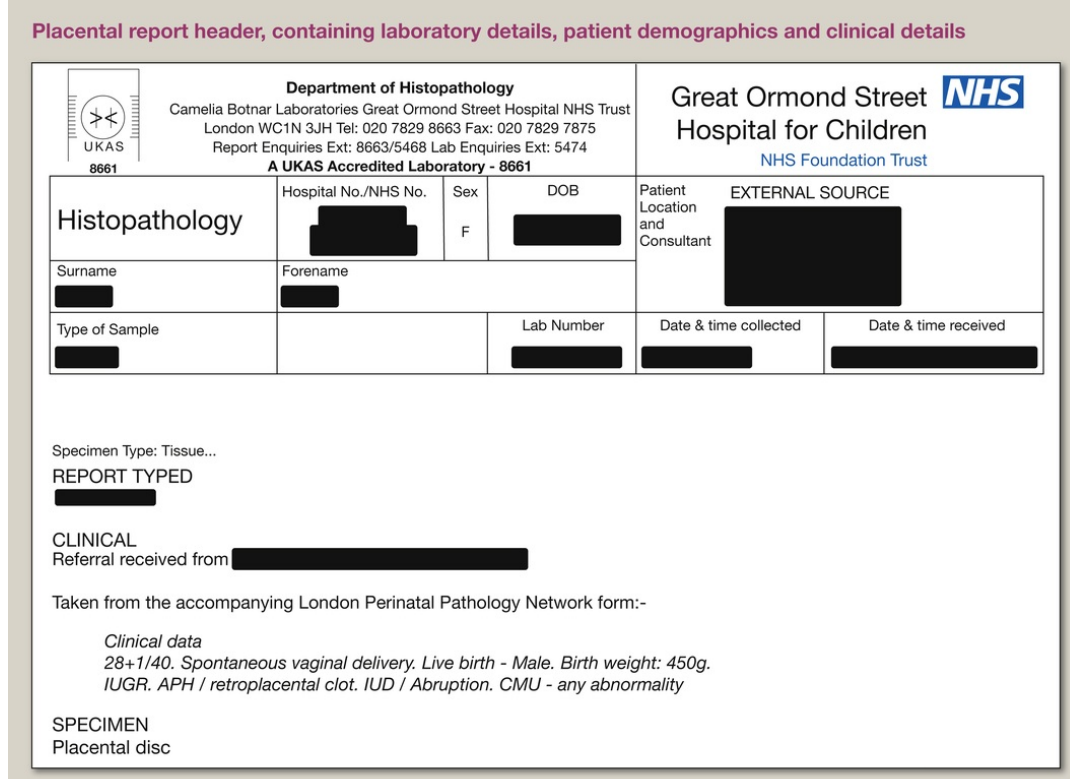

Figure 1

alt-text: Figure 1

\section{The structure of a placental pathology report: macroscopic examination}

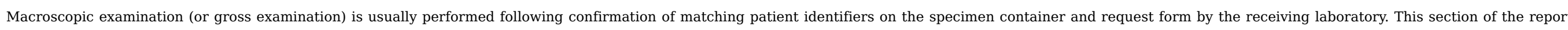

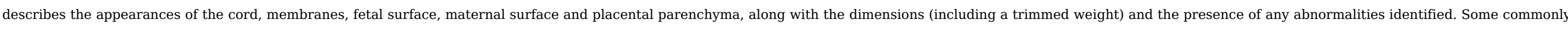
seen macroscopic abnormalities and the related pathologies are summarised in Table 1 below.

Table 1 Possible macroscopic abnormalities at placental examination with associated pathology alt-text: Table 1

\begin{tabular}{|l|l|l}
\hline \multicolumn{2}{|c}{ Macroscopic abnormality } & Related pathology \\
\hline Opaque membranes & Ascending maternal genital tract infection \\
\hline Green, slippery membranes & Intrauterine meconium release \\
\hline Circumvallation of membranes & Chronic abruption sequence or incidental \\
\hline Barber-shop pole cord & Funisitis \\
\hline Two vessel cord (single umbilical artery) & May be associated with fetal anomalies or incidental \\
\hline Marginal or velamentous cord insertion & Haemorrhage, thrombosis, mechanical impingement of cord during labour or incidental \\
\hline Distorted and dilated chorionic plate vessels & Placental mesenchymal dysplasia \\
\hline Organising thrombi within chorionic plate vessels & Fetal vascular malperfusion \\
\hline Clot distorting fetal surface & Sub-amniotic haemorrhage (may be artefactual) & Potential for retained products of conception \\
\hline Maternal surface ragged &
\end{tabular}




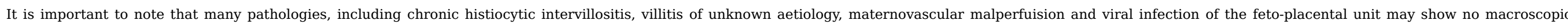

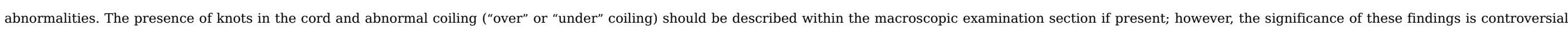
and requires close clinical correlation with the history and any other pathological features.

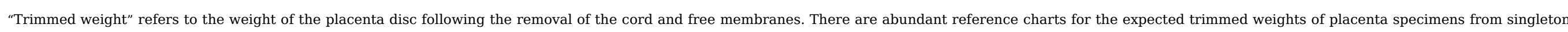

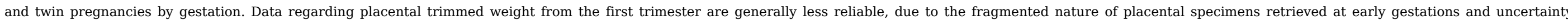

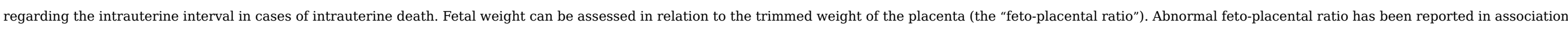
with poor pregnancy outcomes but the overall value of placental weight measurement remains uncertain.

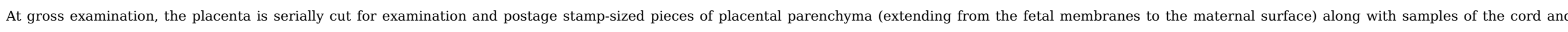

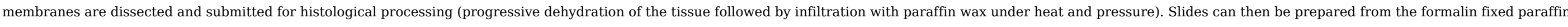

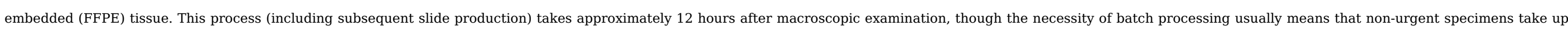
to 24 hours for slides to be created following macroscopic examination.

In the example provided (Figure 2), the trimmed weight is low for the stated gestation (10th centile; $214 \mathrm{~g}$ is $50^{\text {th }}$ centile at 28 weeks).

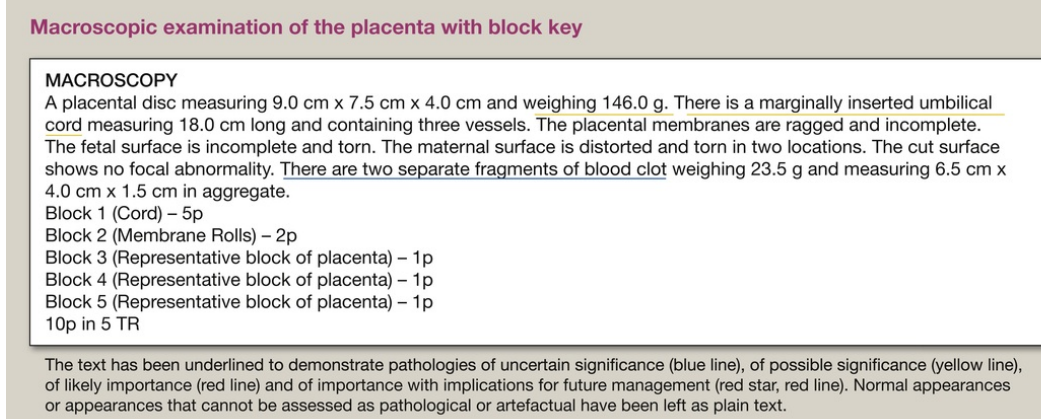

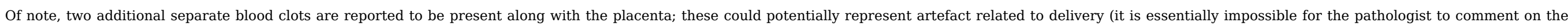
significance of separate clots in isolation). The description of the maternal surface ("torn", "distorted") and fetal membranes ("ragged and incomplete") raises the possibility of retained placental fragments.

\section{The structure of a placental pathology report: microscopic examination}

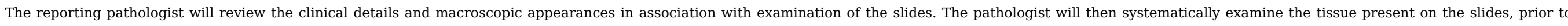

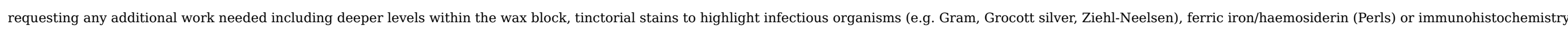
(e.g. CD3 to highlight T-cell aggregates within villi in VUE or some infectious agents (e.g. CMV, toxoplasma). 


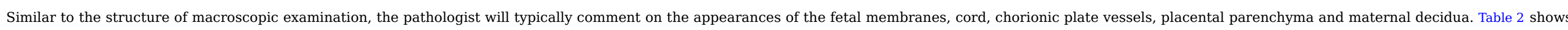
some potential abnormalities identified on microscopy and their significance.

Table 2 Possible microscopic abnormalities at placental examination with associated pathology alt-text: Table 2

\section{Microscopic abnormality}

Neutrophil infiltration of membranes

Haemosiderin within the membranes

Umbilical vein phlebitis/vasculitis/funisitis

Thrombi within chorionic plate vessels/avascular villi

Frequent immature villi after $34 \mathrm{gw}$

Abundant knots with over-mature villi

Lymphocytes within villi with villous damage

Macrophages within maternal space with agglutination of villi

Low density villi with few, thin-calibre terminal villi (over 34 weeks)

Plasma cells within the maternal decidua

Atherosis of maternal spiral arterioles

Sickled maternal erythrocytes

Over-abundance of nucleated fetal erythrocytes in late second to third trimester

\section{Related pathology}

Ascending maternal genital tract infection

Previous premature placental separation/marginal haemorrhage

Ascending maternal genital tract infection with fetal inflammatory response

Fetal vascular occlusion

Delayed villous maturation

Accelerated villous maturation/maternovascular malperfusion

Villitis of unknown aetiology

Chronic histiocytic intervillositis

Distal villous hypoplasia/maternovascular malperfusion

Chronic deciduitis

Maternal vascular malperfusion/vasculopathy

Possible maternal sickle cell trait

Possible fetal hypoxia

In the example provided, the microscopy is structured into sections, reporting on the cord, followed by the extraplacental membranes, chorionic plate, and lastly the placental parenchyma with decidua.

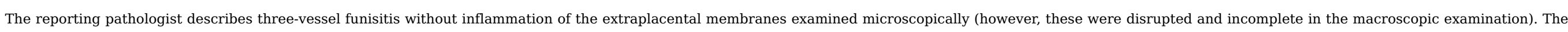
chorionic plate shows acute inflammation. In combination with the funisitis, the description is of ascending maternal genital tract infection with a fetal inflammatory response (Figure 3).

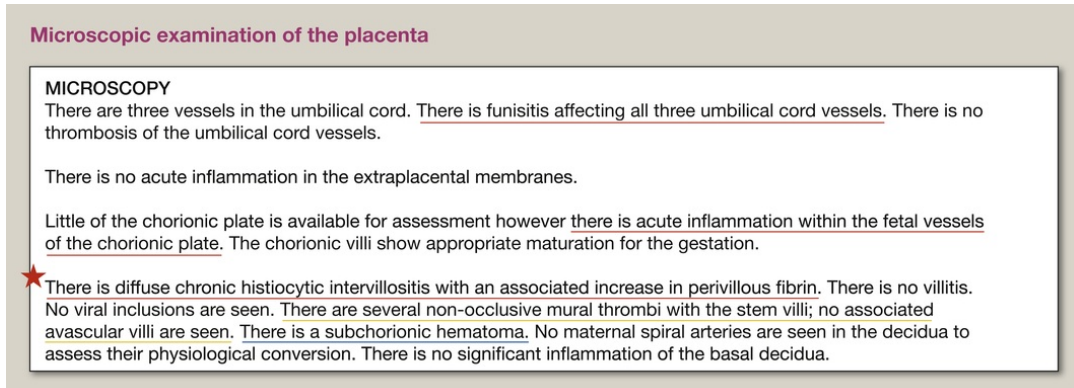

Figure 3

alt-text: Figure 3

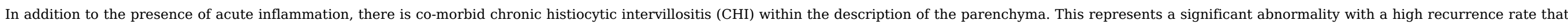
has implications for counselling regarding future pregnancies. 


\section{The structure of a placental pathology report: comment and conclusion}

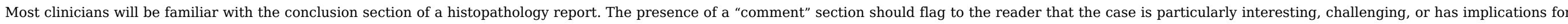

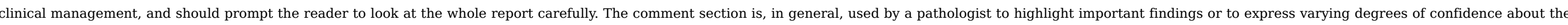
subjective importance of an objective finding. In this case, the pathologist describes the perceived importance of the abnormalities present (Figure 4).

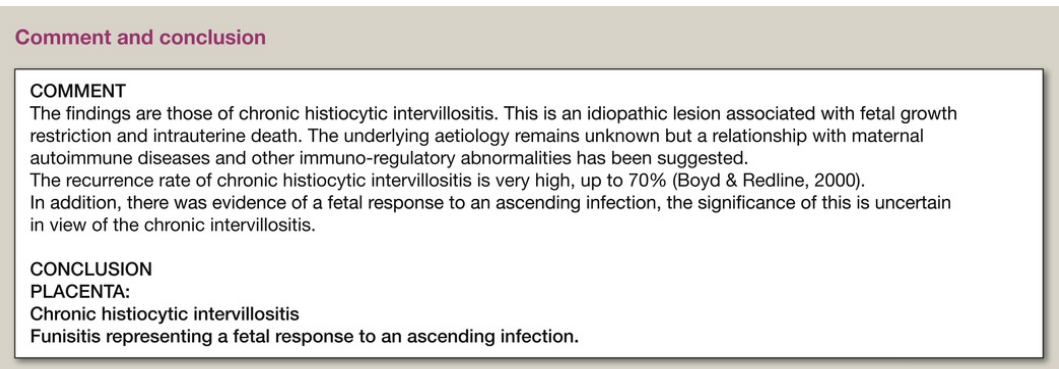

Figure 4

alt-text: Figure 4

\section{Placenta reports: integrating findings and counselling}

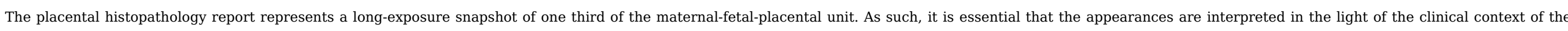

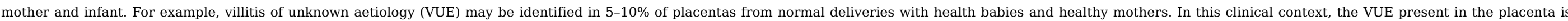

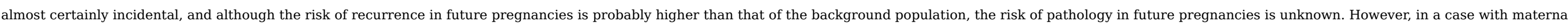

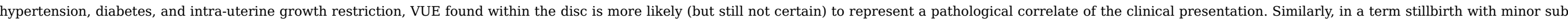

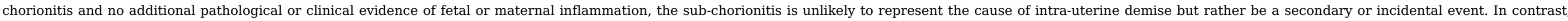

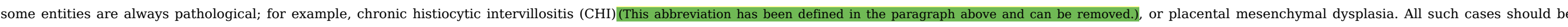
discussed with a fetal medicine specialist to optimise future management.

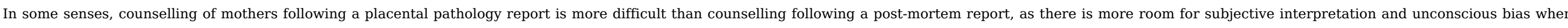

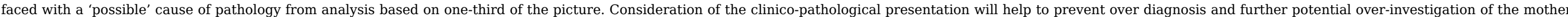
and/or baby for pathology.

The structure of a post-mortem report

The structure of a post-mortem report: clinical details \& supporting information

In general, the types of post-mortem performed by pathologists can be divided by clinical presentation as follows:

1. Intrauterine fetal death (IUFD):

2. Termination of pregnancy (ToP):

3. Peripartum stillbirth/Intrapartum stillbirth

4. Neonatal death

5. Deaths in infancy, including sudden unexpected death in infancy (SUDI) 
Many neonatal deaths, infant deaths and childhood deaths are medicolegal in nature being mandated by the Coroner in the jurisdiction where death occurred, and are beyond the scope of this article

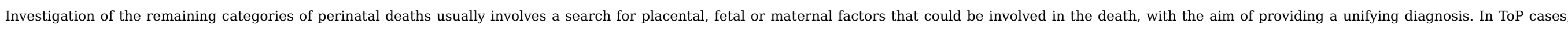

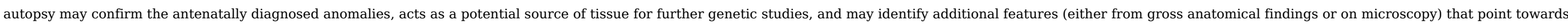

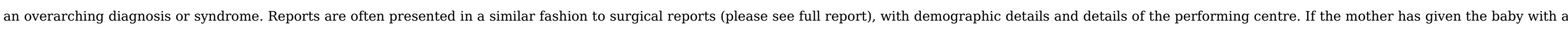

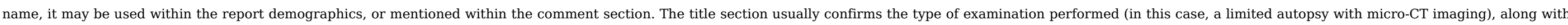
the summary of major pathological findings (Figure 5).

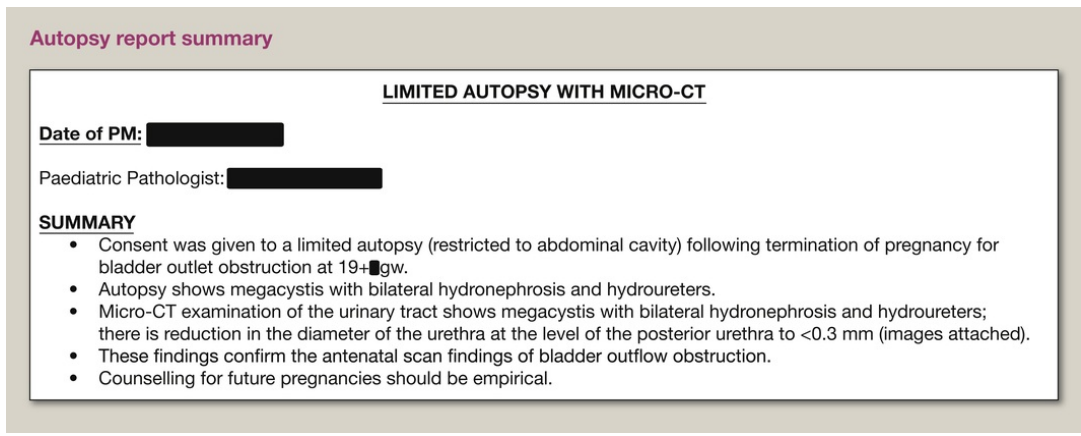

\section{Figure 5}

\section{alt-text: Figure 5}

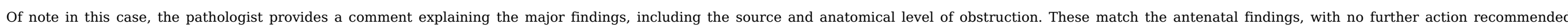

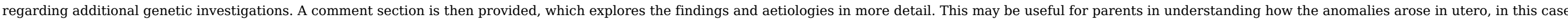
(Figure 6).

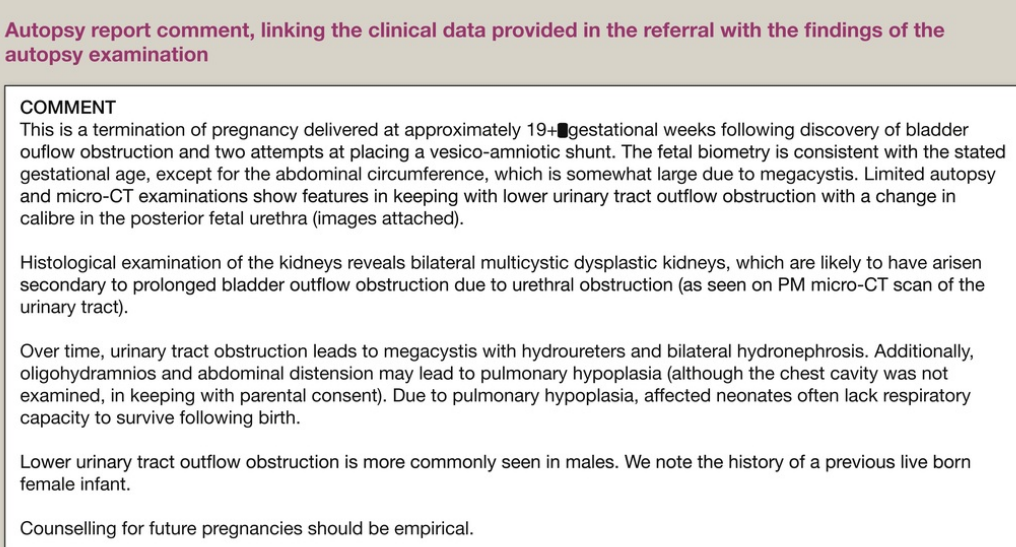




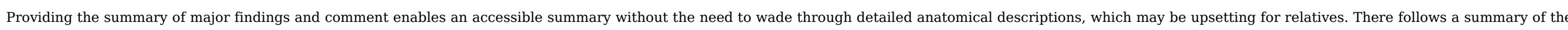

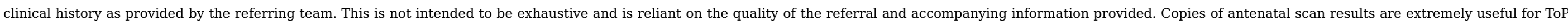
cases and many pathologists will refuse to proceed with an autopsy without reviewing them.

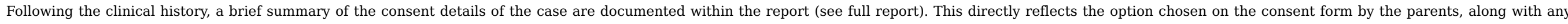

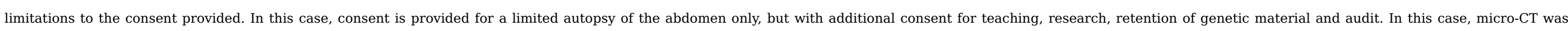
performed as part of direct clinical care with parental consent in order to visualise the level of the urinary tract obstruction.

\section{The structure of a post-mortem report: external examination}

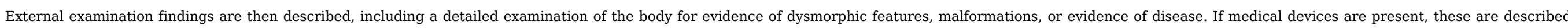

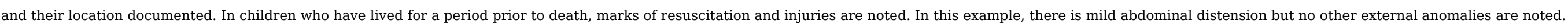

A brief table of potential abnormalities discovered at external examination and related pathologies is provided in Table 3.

Table 3 Possible external abnormalities at autopsy examination with associated pathology

alt-text: Table 3

Macroscopic abnormality (external examination)

Abnormal head shape

Encephalocoele

Cryptophthalmos

Hypertelorism

Broad nasal bridge, low set ears, hypertelorism

Cystic hygroma

Polydactyly

Absent thumb

Chest and abdominal wall defects, externalisation of the heart

Anal atresia

Lower limb abnormalities

Ambiguous genitalia
Possible related pathology

Craniosynostosis

Meckle (Typo identified, this should be "Meckel Gruber syndrome")Gruber syndrome, neural tube defect

Fraser syndrome

Noonan syndrome, Opitz G syndrome

Potter sequence

Monosomy X, Trisomy 21, Noonan syndrome

Ciliopathy, skeletal dysplasia, Trisomy 13

VACTERL association, Fanconi anaemia, Thombocytopaenia-absent radius syndrome

Pentalogy of Cantrell

VACTERL, caudal regression syndrome

Skeletal dysplasia, fibular aplasia spectrum

Denys-Drash syndrome, Lissencephaly

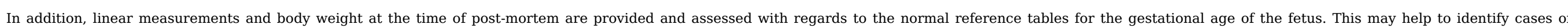

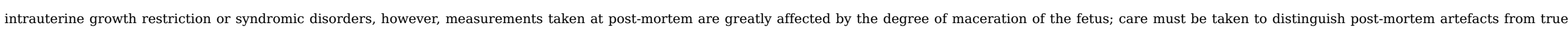
pathology.

\section{The structure of a post-mortem report: post-mortem radiology}

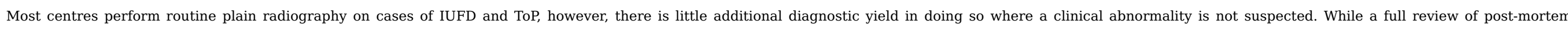

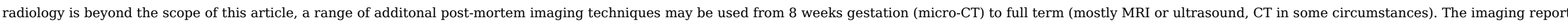
is then integrated into the pathology report. 


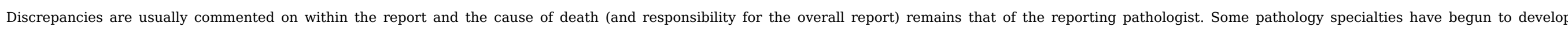

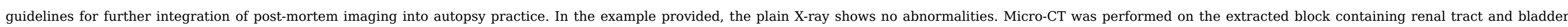
which confirmed the anatomy of the obstruction along with obstructive type cystic changes present in the kidneys.

\section{The structure of a post-mortem report: internal examination}

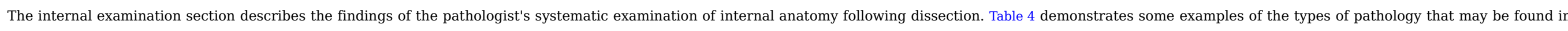
each body system. In addition, the weights of the internal organs are recorded.

Table 4 Possible internal abnormalities at autopsy examination with associated pathology alt-text: Table 4

\begin{tabular}{|c|c|}
\hline Body system & Examples of possible internal abnormalities \\
\hline CNS (brain, spinal cord) & $\begin{array}{l}\text { Neural tube defect, abnormal gyration, ventriculomegaly, intracranial haemorrhage, calcifications, absent corpus callosum, posterior } \\
\text { fossa cyst, vermian hypoplasia }\end{array}$ \\
\hline CVS (Heart, great vessels, peripheral vascular system) & $\begin{array}{l}\text { Abnormal segmental anatomy (atria, ventricular or outflow tract level), abnormal coronary artery anatomy, cardiomyopathy, single } \\
\text { umbilical artery, infarction }\end{array}$ \\
\hline Respiratory (upper and lower respiratory tract, lungs) & Atresia, fistula, pulmonary hypoplasia, diaphragmatic hernia, anatomical isomerism, pleural effusion \\
\hline GI & Atresia, malrotation, perforation, volvulus, abnormal connection, ascites, biliary atresia \\
\hline GU & Cystic renal dysplasia, Renal agenesis/dysgenesis, duplex ureter, megacystis, cloacal anomaly \\
\hline LR & Splenomegaly, lymphadenopathy, cystic hygroma, thymic hypoplasia/aplasia, anatomical abnormality \\
\hline Endocrine & Abnormal anatomy (e.g. annular pancreas), atresia, pituitary/adrenal haemorrhage \\
\hline MSK & Abnormal skeletal or muscle formation, abnormal muscle bulk, fixed flexion deformity \\
\hline
\end{tabular}

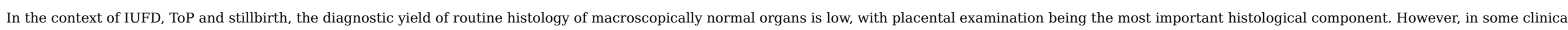

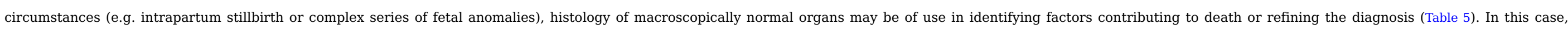

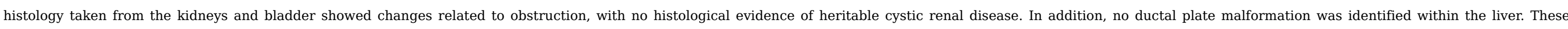

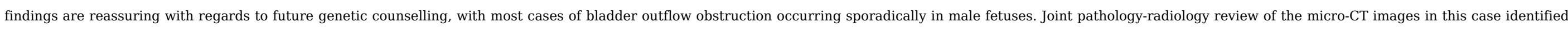
the presence of posterior urethral valves distal to the prostatic urethra.

Table 5 Possible microscopic abnormalities at autopsy examination with associated pathology alt-text: Table 5

$$
\text { Body system }
$$

CNS (brain, spinal cord)

CVS (Heart, great vessels, peripheral vascular system) Respiratory (upper and lower respiratory tract, lungs)

GU

\section{Examples of possible histological abnormalitiesa}

Neuronal migration defect, periventricular leukomalacia, detato-olivary dysplasia, hippocampal sclerosis, hypoxic ischaemic encephalopathy, inflammation, haemorrhage

Myocarditis, abnormal accumulation of material (e.g. glycogen), giant mitochondria, fibrosis, cardiomyopathy

Fetal pneumonia, alveolar capillary dysplasia \pm misalignment of pulmonary veins, bronchial atresia, cystic malformations, lung growth defects, immaturity, interstitial lung disease

Aganglionosis, extra muscle layers, ischaemia, neutrophils within the lumen

Cystic renal disease (including subtype), haemorrhage, glomerular developmental anomalies, nephronophthisis, nephrogenic rests 
a Haemorrhage, infarction, thrombosis, or viral cytopathic changes may be seen in any system.

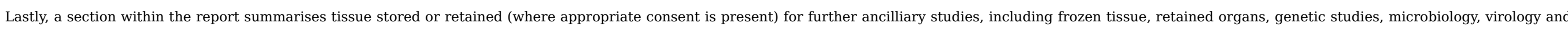
biochemistry.

\section{Post-mortem pathology reports: integration of findings}

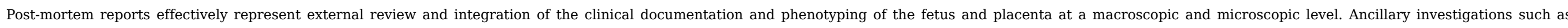

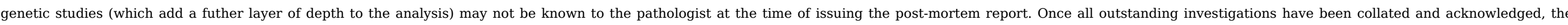

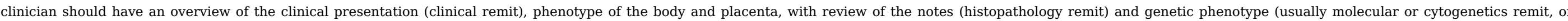

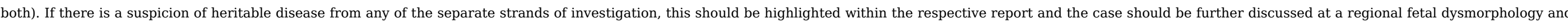

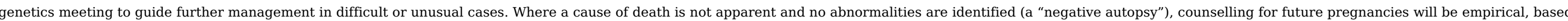

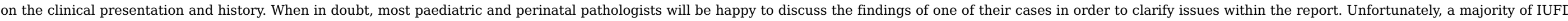

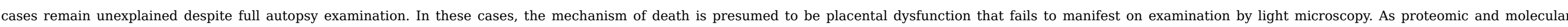
investigations improve and exapnd (Typo identified, the correct wording is "expand")to include formalin fixed tissue, research may help to shed light on the pathogenic mechanisms involved.

\section{Practice points}

- Pathologist interpretation of the clinical significance of objective findings in both placental and post-mortem reports is reliant on both objective findings and adequate clinical information

- Histology of the placenta is the most important investigation in IUFD, providing a cause of death in approximately $20-25 \%$ of cases

- Some placental pathologies have high recurrence rates (CHI, high-grade VUE): others may be incidental

- Counselling for future pregnancies following post-mortem examination should take into account clinical presentation, pathological phenotype, and any genetic or other ancillary findings

\section{Queries and Answers}

Query: Please check the hierarchy of the section headings.

Answer: Checked, these appear correct, thank you.

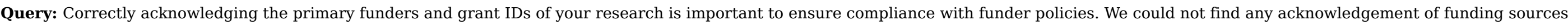
in your text. Is this correct?

Answer: This is correct, I have double-checked this with Prof. Sebire.

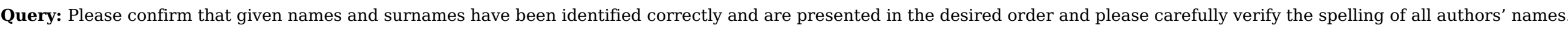

Answer: These are both correct in spelling and layout. I would be grateful if you could indicate that Neil Sebire is the corresponding author. 